\title{
Absolute and relative accelerometer thresholds for determining the association between physical activity and metabolic syndrome in the older adults: The Generation-100 study
}

Nina Zisko ${ }^{1 \dagger}$, Javaid Nauman ${ }^{1 \dagger}$, Silvana Bucher Sandbakk', Nils Petter Aspvik², Øyvind Salvesen ${ }^{3}$, Trude Carlsen $^{4}$, Hallgeir Viken ${ }^{1}$, Jan Erik Ingebrigtsen ${ }^{2}$, Ulrik Wisløff',5 and Dorthe Stensvold1*

\begin{abstract}
Background: When assessing population adherence to physical activity (PA) recommendation using accelerometers, absolute intensity threshold definition is applied despite having limited validity in those with low cardiorespiratory fitness (CRF), who are unable to reach them (e.g older adults). Thus, PA thresholds relative to CRF may be an alternative approach. We compared the proportion of the older adults meeting the PA recommendation when PA is assessed using absolute versus sex-and-CRF-adjusted (relative) accelerometer thresholds and determined the association between relative versus absolute moderate PA (MPA), vigorous PA (VPA) and moderate-to-vigorous PA (MVPA) and metabolic syndrome (MetS).
\end{abstract}

Methods: Cross-sectional study of 509 men and 567 women aged 70-77. Accelerometer assessed MPA, VPA and MVPA were analyzed using absolute and relative thresholds. Meeting the PA-recommendation was defined as amounting $\geq 150 \mathrm{~min} /$ week in MPAMMVPA or $75 \mathrm{~min} /$ week in VPA, respectively. CRF was directly measured as peak oxygen uptake $\left(\mathrm{VO}_{2 \text { peak }}\right)$. MetS was defined as 3 or more of the following: elevated waist circumference, fasting glucose, hypertension, triglycerides, decreased HDL-cholesterol or diabetes, dyslipidemia or hypertension medication.

Results: Higher proportion of the population met the recommendation when PA was assessed with relative compared to absolute thresholds: VPA (72.4\% vs. 1.7\%) and MVPA (75.2\% vs. 33.8\%). Logistic regression analysis revealed that men and women not meeting the relative-MVPA or VPA recommendation had higher likelihood of MetS (Men: MVPA OR: 1.59, 95\% Cl: 1.08-2.33. VPA OR: 1.81, 95\%Cl: 1.23-2.67 and Women: MVPA OR: 2.12, 95\% Cl: 1.36-3.31; VPA OR: 1.95, 95\% Cl: 1. 29-2.95), compared to men and women meeting the relative MVPA or VPA recommendation. There was no significant association between MetS and absolute MVPA, MPA or VPA recommendations in the fully adjusted model.

(Continued on next page)

\footnotetext{
* Correspondence: dorthe.stensvold@ntnu.no

${ }^{\dagger}$ Equal contributors

'The K.G. Jebsen Center of Exercise in Medicine at Department of Circulation and Medical Imaging, Faculty of Medicine and Health Sciences, Norwegian University of Science and Technology, Trondheim, Norway

Full list of author information is available at the end of the article
} 
(Continued from previous page)

Conclusions: The association between meeting/not meeting the PA recommendation and MetS differed with method. Not meeting relative MVPA and VPA recommendation was associated with significantly higher likelihood for presence of MetS. Since relative intensity is part of the current PA recommendation, it should be considered when assessing population PA and associated health risks in the older adults.

Trial registration: Clinical Trial Registration: NCT01931111 (Date of trial registration: July 19, 2013).

Keywords: Actigraph, Oxygen uptake, Aging, $\mathrm{VO}_{2 \text { peak, }}$

\section{Background}

More people die from cardiovascular disease (CVD) than from any other cause [1]. Since physical activity (PA) is important for cardiovascular health, all adults are recommended to perform $\geq 150 \mathrm{~min}$ of moderate or $\geq 75 \mathrm{~min}$ of vigorous PA weekly, or some combination of the two [2]. The PA intensity can be expressed as absolute or relative. Absolute intensity is quantified using work energy expenditure (i.e. metabolic equivalents-of-task or METs), while relative intensity is determined relative to individual cardiorespiratory fitness (CRF) (i.e. peak oxygen uptake or $\left.\mathrm{VO}_{2 \text { peak }}\right)$ and differs for the unfit compared to fit individuals [3].

Accelerometers are often used to objectively assess population adherence to PA recommendation $[4,5]$. Accelerometer output is given in counts [6]. However, the count thresholds used to define moderate-to-vigorous physical activity (MVPA) and assess PA recommendation adherence, are based on absolute intensity and are derived from physical exertion of healthy young to middle-aged adults [7]. These thresholds could have low validity in those with low CRF (i.e. older adults). As the CRF declines with age, it results in changes in relative effort required to perform PA $[8,9]$ and for many unfit older adults, absolute light intensity PA $(<3 \mathrm{METs})$ requires moderate relative effort, while absolute vigorous intensity PA (6-9 METs) is often unattainable [8]. Those not meeting the absolute PA recommendation may be meeting the relative PA recommendation, likely resulting in underestimation of PA-recommendation adherence in this population.

It is estimated that 5.4 million people in the United Kindom would attain or exceed vigorous relative intensity ( $>70 \%$ of $\mathrm{VO}_{2 \text { peak }}$ ) by walking at $\approx 4.8 \mathrm{~km} / \mathrm{h}[10]$. Therefore, even low absolute PA, if performed at high relative intensity, has potential to benefit many by improving CRF, which is a powerful predictor of mortality [11, 10]. However, relative PA assessment in a population is hindered by methodological limitations [12] and until recently, the only available relative thresholds were derived from physical exertion of young to middle-aged healthy adults. Further, methodology associated with application of these thresholds is rather complex, limiting their use to smaller studies [13].

Since PA recommendation is also given in relative intensity [2, 14], it may be valuable, in populations of varying CRF (i.e. older adults) to measure MVPA using recently published relative thresholds derived from physical exertion of the older adults [15]. Furthermore, it is not known if absolute or relative thresholds quantify MVPA that better associates with metabolic syndrome (MetS) [16], which was found to associate with CVDand all-cause mortality in the older adults $[17,18]$ or what role the two components of MVPA, moderate (MPA) and vigorous (VPA) physical activity, play.

The aim of this study, therefore, was to compare the proportion of the older adults meeting relative versus absolute PA recommendation and to determine the method which quantifies PA that better associates with MetS.

\section{Methods}

\section{Study participants}

This study is a part of the Generation 100 study, which aims to investigate the effect of exercise training on morbidity and mortality in the older adults. The Generation 100 study is described elsewhere [19] but briefly: 1567 of 6966 invited inhabitants of Trondheim (Norway), 70-77 years of age, fulfilled the inclusion criteria [19]. For the current study, we excluded participants with incomplete or missing PA $(n=336)$, fasting glucose (fasting time $<8 \mathrm{~h})(n=130)$, and $\mathrm{VO}_{2 \text { peak }}(n=25)$ data (Fig. 1). A total of 1076 (567 women) participants were included in the analyses.

All participants signed informed consent. The study was approved by the Regional Committee for Medical Research Ethics (2013/1609/REK Midt) and complied with the Declaration of Helsinki principles.

\section{Examinations}

All examinations were conducted between August 2012 and June 2013. Detailed protocol is published elsewhere [19]. Briefly, participants were asked to come to the clinic on two separate days.

On day one, blood samples were taken and weight, height, waist-circumference and blood pressure were measured. Information on prescribed medication (hypertension, dyslipidemia and diabetes), alcohol, smoking status, and CVD (myocardial infarction, angina pectoris, heart failure, atrial fibrillation, other heart diseases and stroke) was obtained from a questionnaire [19]. 


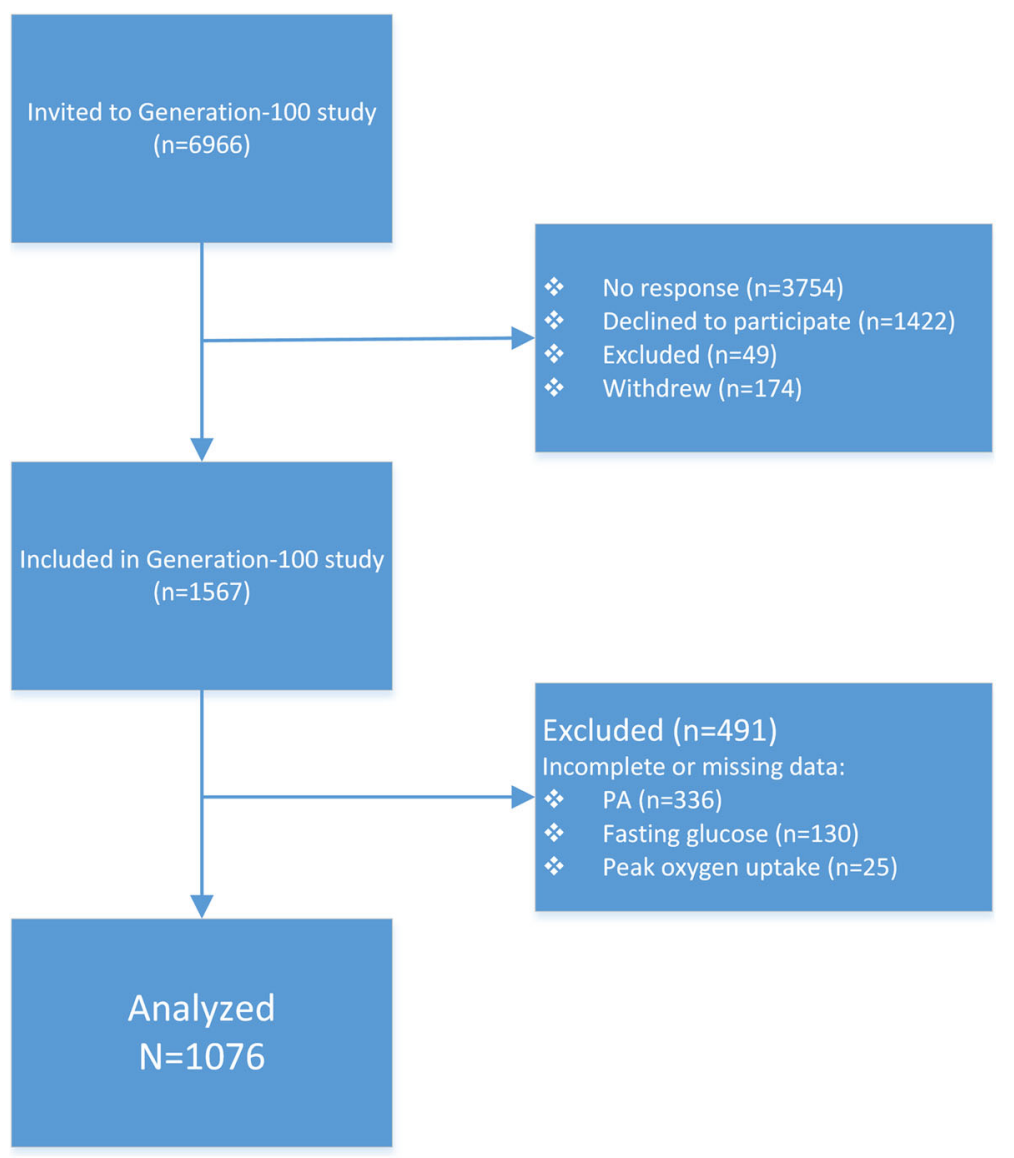

Fig. 1 Study flowchart

On day two, $\mathrm{VO}_{2 \text { peak }}$ was measured using ergospirometry employing an incremental protocol previously described elsewhere [19, 20]. Participants reporting CVD were tested using the American College of Sports Medicine/American Heart Association [21].

All participants were given Actigraph GT3X+ (Actigraph, Pensacola, USA), and were asked to wear it continuously for 7 consecutive days. Actigraph assesses acceleration, and hence PA, in three different axes. While vertical axis (VA) has been most utilized in research, triaxial (VM) motion captures more complex movement $[22,23]$. The VM model was found to better predict relative PA in the older adults than the VA-model and was for that reason used to quantify relative PA in this study [15]. The Actigraph output is given in counts per minute (CPM). The higher the CPM, the higher the estimated PA-intensity [24]. Each sample of data was summed over a 10-s epoch. Data between midnight and 6 am $(6 \mathrm{~h})$ and non wear time were excluded from the analysis. Non wear time was defined as intervals of zero counts lasting at least 60 consecutive minutes, with counts exceeding zero for no more than 2 min [5]. Participants with valid PA data of $\geq 10 \mathrm{~h}$ on $\geq 4$ days were included in the analysis [5]. To quantify PA, the registered accelerometer time was categorized into intensity zones using previously published absolute [7] and relative thresholds [15] and time in different intensity zones was calculated by summing all minutes of PA above the respective thresholds. Briefly, the relative intensity MVPA ( $>62 \%$ of maximum heart rate), MPA (63-76\% of maximum heart rate) and VPA ( $>77 \%$ of maximum heart rate) thresholds used in the current study were derived from and for the Generation 100 population [15]. To establish the relative thresholds, subjects from the Generation 100 study, wearing an Actigraph GT3+ model, walked/run on the treadmill while having submaximal and maximal oxygen uptake measured [15]. Relationship between maximum oxygen uptake $\%$, maximum heart rate \%, VM-CPM and sex was established using a mixed regression model. Detailed protocol of relative threshold derivation is published elsewhere [15]. The Freedson absolute intensity thresholds applied in the current study are an established method used to examine PA recommendation adherence [7]. Detailed protocol of Freedson absolute threshold derivation is published elsewhere [7]. All MVPA and MPA was analyzed in 10min-bouts (with up to 2-min interruption allowance) and 
VPA in 5-min-bouts (with up to 1-min interruption allowance). All PA was wear-time adjusted by multiplying recorded PA time by $1080 \mathrm{~min}(24 \mathrm{~h}$ minus $6 \mathrm{~h}$ from midnight to $6 \mathrm{am}$ ) and dividing it by wear-time in minutes. PA analysis was done using Actilife 6.11 .5 (Actigraph, Pensacola, USA).

\section{Data and statistical analysis}

Descriptive data is presented as mean \pm standard deviation for continuous and percentages for categorical variables. To test parameter differences between sexes of continuous variables, $t$-test was used. The chi square test was used to assess sex differences between categorical variables. Presence of at least three of the following five risk factors was defined as MetS: increased waist-circumference ( $\geq 80 \mathrm{~cm}$ in women and $\geq 94 \mathrm{~cm}$ in men); increased blood pressure (systolic $\geq 130 \mathrm{mmHg}$ and/or diastolic $\geq 85 \mathrm{mmHg}$ ) or drug treatment for hypertension; decreased HDLcholesterol $\left(<1.3 \mathrm{mmol} \cdot \mathrm{L}^{-1}\right.$ in women and $<1.0 \mathrm{mmol} \cdot \mathrm{L}^{-1}$ in men) or drug treatment for dyslipidemia; increased triglycerides $\left(\geq 1.7 \mathrm{mmol} \cdot \mathrm{L}^{-1}\right)$ or drug treatment for dyslipidemia; and increased fasting glucose $\left(\geq 5.6 \mathrm{mmol} \cdot \mathrm{L}^{-1}\right)$ or drug treatment for diabetes [16]. Meeting the PA recommendations was classified based on the current American College of Sports Medicine/American Heart Association PA recommendations for older adults [2]. Henceforth, an accumulated time of $\geq 21.43 \mathrm{~min} /$ day (i.e. $\geq 150 \mathrm{~min} /$ week) spent at MPA and MVPA (in bouts of at least $10 \mathrm{~min}$ ) and $\geq 10.71 \mathrm{~min} /$ day (i.e. $\geq 75 \mathrm{~min} /$ week) spent at VPA (in bouts of at least $5 \mathrm{~min}$ ) was considered as meeting the current PA recommendations. The minutes spent in bouts of absolute and relative MVPA and MPA were summed up, and then dichotomized (below/meeting recommendation), so that those with $\geq 150$ weekly minutes $(21.4 \mathrm{~min} /$ day $)$ were considered to have met the PA-recommendation. The minutes spent in bouts of VPA were summed up and dichotomized (below/meeting recommendation), so that those amounting $\geq 75$ weekly minutes (or $10.7 \mathrm{~min} /$ day) were considered to have met the PA-recommendation. Logistic regression analyses were used to estimate the association between fulfillment of relative and absolute PA recommendation (i.e. below/meeting recommendation) and prevalence of MetS stratified by sex. Results are presented as odds ratios, and 95\% confidence intervals (CI) were used to assess precision of estimates. Study participants that were meeting the PA recommendation were used as a reference in the logistic regression analysis. The basic PA model (model 1) was adjusted for age, with additional adjustment for smoking status, alcohol consumption, and history of CVD (model 2), with absolute PA additionally adjusted for CRF (model 3).

To test the robustness of our results, we performed a sensitivity analysis in those without (869 participants) and those with CVD (207 participants) in our cohort. To determine if the model improved the fit, a log-likelihood-ratio test was used. To compare the maximum likelihood models combining fit and complexity, we used the BayesianInformation-Criterion (BIC) and Akaike-InformationCriterion (AIC) [25]. The smaller information criterion (AIC or BIC) denotes the model that fits the data better. All statistical tests were two-tailed and were performed using Stata (version 13.1 StataCorp.). The results were considered statistically significant if the $p$-value was less than 0.05 .

\section{Results}

Characteristics of study participants, clinical measurements and questionnaires

The characteristics of study participant are shown in Table 1 . The mean age was $72.5 \pm 2.1$ years. In total, $31.9 \%$ of our participants received drug treatment for hypertension, $6.0 \%$ for diabetes, and $8.9 \%$ for dyslipidemia. Eleven percent were obese $(\mathrm{BMI}>30)$. Elevated waist circumference was the most predominant risk factor in both men and women with $77.9 \%$ and $77.4 \%$ prevalence, respectively. Second most predominant risk factor was elevated blood pressure and/or treatment for hypertension with $68.2 \%$ and $66.3 \%$ prevalence for men and women, respectively. Of the study sample, $38 \%$ were categorized as having MetS.

No sex differences were observed for triglycerides, HDL-cholesterol, diastolic blood pressure, or in time spent in absolute MVPA. Men had higher waistcircumference, fasting glucose, history of CVD, alcohol consumption and $\mathrm{VO}_{2 \text { peak }}$. Women spent more time in relative MVPA.

\section{Relative versus absolute intensity physical activity}

Average accelerometer wear time was $16.1 \pm 1.1 \mathrm{~h}$ per day. Four, five and six or more valid days of PA data were available for $1.4 \%, 7.2 \%$ and $91.4 \%$ of the participants, respectively. Average time in bouts of absolute MVPA for men and women was $19.5 \pm 21.4$, and $18.3 \pm 18.8 \mathrm{~min}$ per day, respectively, while time spent in relative MVPA bouts was $33.9 \pm 27.3$ and $53.1 \pm 38.9$ min per day for men and women, respectively. The proportion of men and women meeting the absolute MVPA recommendation did not differ, while a difference between sexes was observed with relative MVPA recommendation. Significantly higher proportion $(40 \%)$ of the population met the relative versus absolute MVPA recommendation. Majority of women (82.2\%) amounted $\geq 150$ weekly minutes in relative MVPA, with only $33.6 \%$ doing so in absolute MVPA. Higher proportion of men (33\%) met the relative MVPA compared to absolute MVPA recommendation. Men and women spent significantly more time in relative compared to absolute VPA and significantly higher proportion of the population met the relative compared to absolute VPA recommendation, while significantly more time was 
Table 1 Characterization of 509 men and 567 women aged 70-77 years participating in the study

\begin{tabular}{|c|c|c|c|}
\hline Characteristics & $\begin{array}{l}\text { All } \\
(n=1076)\end{array}$ & $\begin{array}{l}\text { Men } \\
(n=509)\end{array}$ & $\begin{array}{l}\text { Women } \\
(n=567)\end{array}$ \\
\hline Age & $72.5 \pm 2.1$ & $72.4 \pm 2.1$ & $72.5 \pm 2.1$ \\
\hline MetS $^{\mathrm{a}},(\%)$ & 38.0 & $42.8^{*}$ & 34.2 \\
\hline Waist circumference (cm) & $89.3 \pm 10$ & $88.3 \pm 10.7^{*}$ & $91.0 \pm 10.8$ \\
\hline Elevated waist circumference, (\%) & 80.5 & 77.9 & 77.4 \\
\hline Triglycerides $\left(\mathrm{mmol} \cdot \mathrm{L}^{-1}\right)$ & $1.13 \pm 0.54$ & $1.16 \pm 0.57^{*}$ & $1.10 \pm 0.52$ \\
\hline Elevated triglycerides or drug treatment, (\%) & 20.6 & 24.8 & 16.9 \\
\hline HDL-cholesterol $\left(\mathrm{mmol}^{\circ} \mathrm{L}^{-1}\right)$ & $1.74 \pm 0.50$ & $1.56 \pm 0.44^{*}$ & $1.90 \pm 0.50$ \\
\hline Reduced HDL-cholesterol, (\%) & 14.7 & 16.1 & 13.5 \\
\hline \multicolumn{4}{|l|}{ Blood pressure (mmHg) } \\
\hline Systolic & $133 \pm 1$ & $132 \pm 16$ & $134 \pm 19$ \\
\hline Diastolic & $75 \pm 9$ & $77 \pm 9^{*}$ & $73 \pm 9$ \\
\hline Elevated blood pressure or drug treatment (\%) & 67.2 & 68.2 & 66.3 \\
\hline Fasting glucose $\left(\mathrm{mg} \cdot \mathrm{dL}^{-1}\right)$ & $5.67 \pm 0.88$ & $5.85 \pm 0.98^{*}$ & $5.51 \pm 0.77$ \\
\hline Elevated fasting glucose or drug treatment (\%) & 52.7 & 61.4 & 44.6 \\
\hline History of cardiovascular disease ${ }^{\mathrm{b}}(\%)$ & 19.2 & $25.9^{*}$ & 13.2 \\
\hline Currently smoking (\%) & 7.6 & 8.3 & 7.0 \\
\hline Alcohol use (units.week ${ }^{-1}$ ) & $3.5 \pm 3.7$ & $4.2 \pm 4.2^{*}$ & $2.9 \pm 2.9$ \\
\hline $\begin{array}{l}\text { Absolute intensity moderate- to vigorous physical } \\
\text { activity }\left(\min \cdot \text { day }^{-1}\right)\end{array}$ & $18.8 \pm 20.1$ & $19.5 \pm 21.4$ & $18.3 \pm 18.8$ \\
\hline $\begin{array}{l}\text { Meeting absolute intensity moderate-to-vigorous } \\
\text { physical activity recommendation }(\%)^{c}\end{array}$ & 33.8 & 33.9 & 33.6 \\
\hline $\begin{array}{l}\text { Relative intensity moderate- to vigorous physical } \\
\text { activity (min.day }{ }^{-1} \text { ) }\end{array}$ & $44.0 \pm 35.2$ & $33.9 \pm 27.3^{*}$ & $53.1 \pm 38.9$ \\
\hline $\begin{array}{l}\text { Meeting relative moderate- to vigorous intensity } \\
\text { physical activity recommendation }(\%)^{c}\end{array}$ & 75.2 & $67.3^{*}$ & 82.2 \\
\hline Absolute intensity moderate physical activity $\left(\min \cdot\right.$ day $^{-1}$ ) & $16.8 \pm 18.2$ & $16.2 \pm 18.3$ & $17.3 \pm 18.1$ \\
\hline $\begin{array}{l}\text { Meeting absolute moderate intensity physical activity } \\
\text { recommendation }(\%)^{c}\end{array}$ & 30.4 & 28.6 & 32.5 \\
\hline Relative intensity moderate physical activity $\left(\mathrm{min} \cdot \mathrm{day}^{-1}\right.$ ) & $2.6 \pm 9.1$ & $1.8 \pm 6.3$ & $3.3 \pm 11.1$ \\
\hline $\begin{array}{l}\text { Meeting relative intensity moderate intensity physical } \\
\text { activity recommendation }(\%)^{c}\end{array}$ & 3.4 & $1.2^{*}$ & 5.6 \\
\hline Absolute intensity vigorous physical activity $\left(\mathrm{min} \cdot \mathrm{day}^{-1}\right.$ ) & $0.8 \pm 3.7$ & $1.4 \pm 5.2^{*}$ & $0.1 \pm 0.8$ \\
\hline $\begin{array}{l}\text { Meeting absolute vigorous intensity physical activity } \\
\text { recommendation }(\%)^{\mathrm{d}}\end{array}$ & 1.7 & $3.4^{*}$ & 0.2 \\
\hline Relative intensity vigorous physical activity $\left(\min \cdot\right.$ day $^{-1}$ ) & $27.0 \pm 23.2$ & $24.1 \pm 22.6^{*}$ & $29.8 \pm 23.4$ \\
\hline $\begin{array}{l}\text { Meeting relative vigorous intensity physical activity } \\
\text { recommendation }(\%)^{d}\end{array}$ & 72.4 & $67.1^{*}$ & 77.4 \\
\hline Peak oxygen uptake $\left(\mathrm{mL} \cdot \mathrm{kg}^{-1} \cdot \mathrm{min}^{-1}\right)$ & $28.9 \pm 6.6$ & $31.7 \pm 6.7^{*}$ & $26.4 \pm 5.1$ \\
\hline
\end{tabular}

Numbers are means \pm standard deviation unless otherwise specified

*Statistically significant differences between sexes $(p<0.05)$

${ }^{a}$ MetS: Metabolic syndrome risk factor clustering was defined as the presence of at least 3 of the following 5 risk factors: elevated waist circumference (being $\geq 80 \mathrm{~cm}$ in women and $\geq 94 \mathrm{~cm}$ in men); elevated triglycerides (being $\geq 1.7 \mathrm{mmol} \cdot \mathrm{L}-1$ ); reduced HDL-cholesterol (being $\leq 1.3 \mathrm{mmol} \cdot \mathrm{L}-1$ in women and $\leq 1.0 \mathrm{mmol} \cdot \mathrm{L}-1$ in men); elevated blood pressure (systolic $\geq 130 \mathrm{mmHg}$ and/or diastolic $\geq 85 \mathrm{mmHg}$ ); and elevated fasting glucose (being $\geq 100 \mathrm{mg} \cdot \mathrm{dL}-1$ ).

${ }^{b}$ History of CVD was determined with current or former prevalence of myocardial infarction, angina pectoris, heart failure, atrial fibrillation, other heart diseases and/or stroke.

${ }^{\mathrm{C}}$ Having $\geq 150$ min per week moderate to vigorous or moderate intensity in bouts of at least 10 min $=$ meeting absolute or relative moderate to vigorous or moderate PA recommendation

${ }^{\mathrm{d}}$ Having $\geq 75 \mathrm{~min}$ per week in bouts of at least $5 \mathrm{~min}=$ meeting absolute or relative vigorous PA recommendation 
spent in absolute compared to relative MPA and higher proportion of the population met absolute versus relative MPA recommendation (Table 1).

\section{Metabolic syndrome}

Not meeting the relative MVPA recommendation was associated with more than twofold (OR: 2.12, 95\% CI: 1.36-3.31) and 59\% (OR: 1.59, 95\% CI: 1.08-2.33) higher likelihood for having MetS in women and men, respectively, when compared to meeting the relative MVPArecommendation (Table 2). Similarly, not meeting the relative VPA recommendation was associated with $81 \%$ (OR: 1.81, 95\% CI: 1.23-2.67) and 95\% (OR: 1.95, 95\%CI: 1.29-2.95) higher likelihood for having MetS in men and women, respectively. There was no significant association between MetS and absolute MVPA, MPA or VPA recommendations in the fully adjusted model (Table 3, Fig. 2).

The log-likelihood-test was significant $(p<0.001)$, and the fully adjusted model for relative PA was a better fit on the basis of lower AIC and BIC values (absolute MVPA: AIC: 1424.3, BIC: 1434.2 versus relative MVPA: AIC: 1409.89 , BIC: 1419.852). The sensitivity analysis of those without CVD produced similar results, indicating 69\% (OR: 1.69, 95\% CI: 1.21-2.36, Additional file 1: Table S1) higher likelihood for MetS in those not meeting the relative MVPA recommendation and 88\% (OR: 1.88, 95\% CI: 1.36-2.60, Additional file 1: Table S1) higher likelihood of MetS in those not meeting the relative VPA recommendation. In those reporting CVD, not meeting the relative MVPA recommendation was associated with more than twofold higher likelihood (OR: 2.30, 95\%CI: $1.23-4.31$, Table 1) of MetS and 85\% (OR: 1.85 95\%CI: 1.02-3.36, Additional file 1: Table S1) higher likelihood of MetS in those not meeting the relative VPA recommendation. No significant association was observed in those with or without CVD not meeting the absolute MVPA recommendation and MetS (Additional file 1: Table S2).

\section{Discussion}

The main finding of the current study was that a significantly higher proportion of our older adults met the relative versus absolute PA recommendation. Those below the relative PA recommendation were more likely to have increased prevalence of MetS compared to those below the absolute PA recommendation. Importantly, not meeting the relative VPA recommendation was associated with higher likelihood of MetS, while no such association was observed in those below the absolute MPA, VPA or MVPA recommendation.

To our knowledge, this is the first study utilizing accelerometers to objectively assess relative PA in a large population of older adults using thresholds stratified for sex and CRF, and investigating how the relative MVPA and its two components, MPA and VPA, associate with MetS. As individuals age, their CRF declines, producing a change in the relative intensity of effort required for PA $[8,9]$. Many individuals with low CRF, including the older adults, require moderate relative effort for low absolute PA and can rarely reach absolute MPA, MVPA or VPA [8].

Table 2 Adjusted odds ratios (OR; 95\% confidence interval) for the prevalence of MetS according to meeting or not meeting physical activity measured objectively using relative intensity accelerometer thresholds among 509 men and 567 men aged 70-77 years

\begin{tabular}{|c|c|c|c|c|c|c|c|c|}
\hline & \multicolumn{4}{|l|}{ Men } & \multicolumn{4}{|c|}{ Women } \\
\hline & \multicolumn{2}{|c|}{$\overline{\text { MetS }^{c}}$} & \multirow[b]{2}{*}{ OR $(95 \% C l)^{a}$} & \multirow[b]{2}{*}{ OR $(95 \% \mathrm{Cl})^{\mathrm{b}}$} & \multicolumn{2}{|c|}{$\overline{\text { MetS }^{c}}$} & \multirow[b]{2}{*}{ OR $(95 \% C l)^{a}$} & \multirow[b]{2}{*}{ OR $(95 \% \mathrm{Cl})^{\mathrm{b}}$} \\
\hline & $\overline{\text { Yes }}$ & $\overline{\mathrm{No}}$ & & & $\overline{Y e s}$ & $\overline{\mathrm{No}}$ & & \\
\hline \multicolumn{9}{|c|}{ Relative MVPA ${ }^{d}$ recommendation } \\
\hline Below & 86 & 80 & $1.74(1.19-2.53)$ & $1.59(1.08-2.33)$ & 49 & 52 & $2.15(1.39-3.33)$ & $2.12(1.36-3.31)$ \\
\hline Meeting & 132 & 211 & 1.00 (Reference) & 1.00 (Reference) & 142 & 324 & 1.00 (Reference) & 1.00 (Reference) \\
\hline \multicolumn{9}{|c|}{ Relative MPA recommendation } \\
\hline Below & 215 & 287 & $1.00(0.22-4.54)$ & $0.87(0.18-4.09)$ & 182 & 355 & $1.19(0.53-2.66)$ & $1.11(0.49-2.51)$ \\
\hline Meeting & 3 & 4 & 1.00 (Reference) & 1.00 (Reference) & 9 & 21 & 1.00 (Reference) & 1.00 (Reference) \\
\hline \multicolumn{9}{|c|}{ Relative VPA ${ }^{e}$ recommendation } \\
\hline Below & 91 & 76 & $2.02(1.39-2.95)$ & $1.81(1.23-2.67)$ & 57 & 67 & $1.96(1.30-2.95)$ & $1.95(1.29-2.95)$ \\
\hline Meeting & 127 & 215 & 1.00 (Reference) & 1.00 (Reference) & 134 & 309 & 1.00 (Reference) & 1.00 (Reference) \\
\hline
\end{tabular}

Cl confidence interval.

${ }^{\text {a }}$ Model 1: Adjusted for age.

${ }^{\mathrm{b}}$ Model 2: Adjusted for age, smoking status, alcohol consumption, history of cardiovascular disease.

${ }^{\mathrm{C}}$ MetS: Metabolic syndrome risk factor clustering was defined as the presence of at least 3 of the following 5 risk factors: elevated waist circumference (being

$\geq 80 \mathrm{~cm}$ in women and $\geq 94 \mathrm{~cm}$ in men); elevated triglycerides (being $\geq 1.7 \mathrm{mmol} \cdot \mathrm{L}-1$ ); reduced HDL-cholesterol (being $\leq 1.3 \mathrm{mmol} \cdot \mathrm{L}-1$ in women and $\leq 1.0 \mathrm{mmol} \cdot \mathrm{L}-1$

in men); elevated blood pressure (systolic $\geq 130 \mathrm{mmHg}$ and/or diastolic $\geq 85 \mathrm{mmHg}$ ); and elevated fasting glucose (being $\geq 100 \mathrm{mg} \cdot \mathrm{dL}-1$ ).

d bouts of at least $10 \mathrm{~min}$.

ebouts of at least $5 \mathrm{~min}$. 
Table 3 Adjusted odds ratios (OR; 95\% confidence interval) for the prevalence of MetS according to meeting or not meeting physical activity measured objectively using absolute intensity accelerometer thresholds among 509 men and 567 men aged 70-77 years

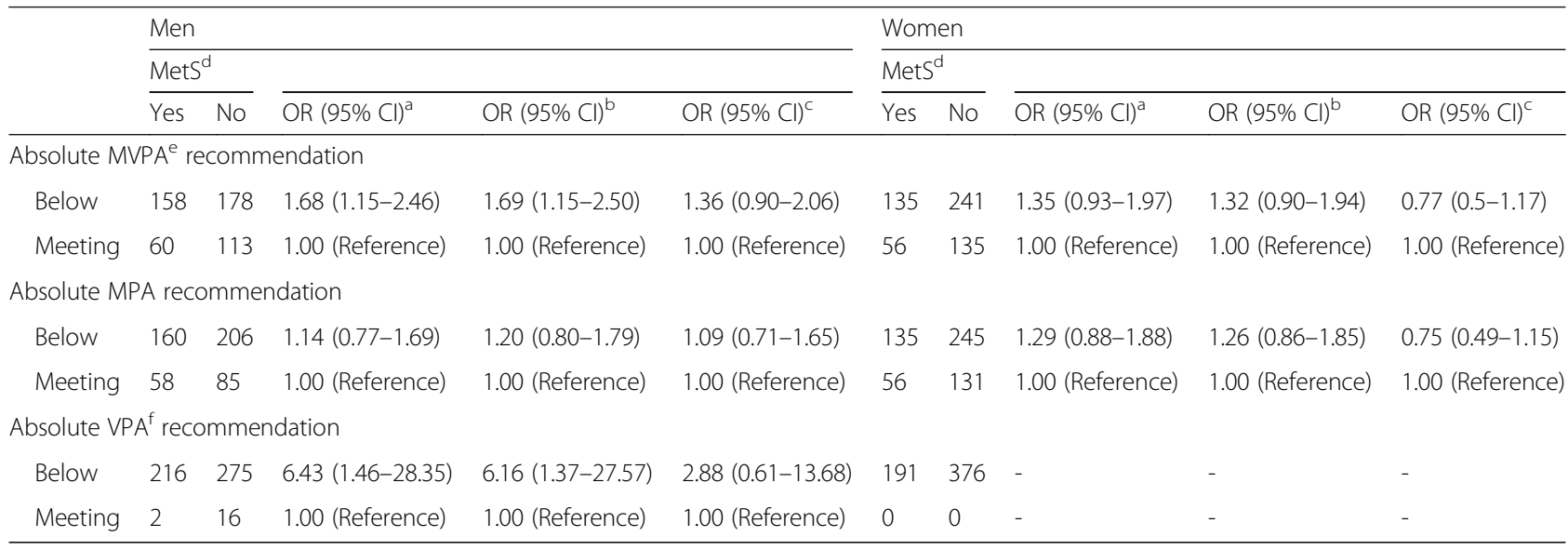

Cl confidence interval.

${ }^{a}$ Model 1: Adjusted for age.

${ }^{\mathrm{b}}$ Model 2: Adjusted for age, smoking status, alcohol consumption, history of cardiovascular disease.

'Model 3: Adjusted for age, smoking status, alcohol consumption, history of cardiovascular disease and cardiorespiratory fitness (measured as VO2peak).

dMetS: Metabolic syndrome was defined as the presence of at least 3 of the following 5 risk factors: elevated waist circumference (being $\geq 80 \mathrm{~cm}$ in women and $\geq 94 \mathrm{~cm}$ in men); elevated triglycerides (being $\geq 1.7 \mathrm{mmol} \cdot \mathrm{L}-1$ ); reduced HDL-cholesterol (being $\leq 1.3 \mathrm{mmol} \cdot \mathrm{L}-1$ in women and $\leq 1.0 \mathrm{mmol} \cdot \mathrm{L}-1$ in men); elevated blood pressure (systolic $\geq 130 \mathrm{mmHg}$ and/or diastolic $\geq 85 \mathrm{mmHg}$ ); and elevated fasting glucose (being $\geq 100 \mathrm{mg} \cdot \mathrm{dL}-1$ ).

ebouts of at least $10 \mathrm{~min}$.

fbouts of at least $5 \mathrm{~min}$.

Previous studies showed accelerometer thresholds to vary with age, with older individuals having lower accelerometer count output. This difference was ascribed to variation in CRF, which stressed the importance of relative intensity when assessing PA in populations of different ages and CRF levels [26]. Ozemek et al. were the first to illustrate the importance of CRF in accelerometer PA assessment when they showed an unfit younger individual to have a significantly lower MVPA threshold compared to a more fit older individual [13]. Notably, they found the MVPA counts to be significantly correlated to CRF, explaining ca. $30 \%$ of the variability, while only ca. $1 \%$ could be explained by age and BMI. However, Ozemek's approach to relative PA assessment is based on physical exertion of younger adults and requires complex methodology, making its use cumbersome in larger studies [13]. In our study we utilized thresholds derived from physical exertion of older adults to quantify relative PA [15]. These thresholds are adjusted for CRF and sex and are feasible to use in large studies [15].

Higher proportion of our participants met the absolute MVPA recommendation compared to other studies on older Norwegians [27-29]. This difference could be ascribed to fewer study participants [27-29], wider age interval [27-29], and different methodology [27-29] of these studies. For instance, these studies utilized the Troiano [27-29], while we used the Freedson threshold [27-29] for absolute MVPA assessment and they applied

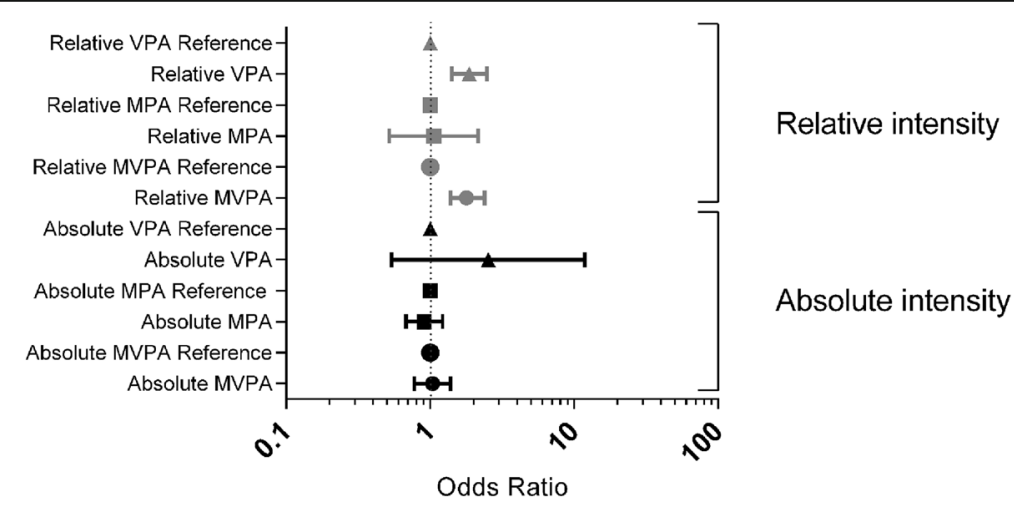

Fig. 2 Adjusted odds ratio for the prevalence of MetS according to adherence to absolute versus relative intensity PA-recommendation among 1076 men and women aged 70-77 years. Study participants that were meeting the PA-recommendation were used as a reference in the logistic regression analysis. The model for relative and absolute PA was adjusted for age, smoking status, alcohol consumption and history of CVD with absolute PA model additionally adjusted for CRF 
an earlier (30 min of daily MVPA) recommendation, while we used the current Norwegian PA recommendation [27-29]. Notably, by applying the earlier PA recommendation in a preliminary analysis of our study, we obtained a similar result, with $23 \%$ of our population meeting the absolute MVPA recommendation.

The lack of association between absolute PA recommendation adherence and MetS in the current study may be attributable to absolute thresholds being too high for this population. Some older adults may have difficulties reaching absolute thresholds and as a result fail to meet the absolute PA recommendation. In fact, results of our absolute VPA analysis show that out of 509 men and 567 women included in our study, only two individuals, both men, managed to meet the absolute VPA recommendation. One could argue that expecting unfit individuals, such as some older adults, to accumulate sufficient time at an absolute intensity higher than their maximal capacity (e.g. $\left.\mathrm{VO}_{2 \text { peak }} \sim 3 \mathrm{METs}\right)$ in order to meet the PA recommendation is unrealistic. Relative thresholds, on the other hand, are individualized in terms of CRF and sex. Indeed, it has been shown that the least fit individuals from the Generation 100 cohort are also least likely to adhere to both absolute and relative PA recommendation compared to the moderately fit and highly fit individuals [30]. Approximately $30 \%$ of our population fail to meet the relative PA recommendation. This is important information as it may allow researchers to identify the least active individuals when designing strategies to increase PA participation in older adults. Our results encourage researchers to consider PA intensity in both relative and absolute terms when utilizing accelerometers in assessment of PA, especially in populations with varying degrees of fitness and physical functionality. Thus, meeting the relative PA recommendation for older adults may not only prove a realistically achievable goal, but may be beneficial for health.

The strength of our study is that it uses objective measures of absolute and relative PA, and relates them to objectively measured health indicator in a large sample of older adults. Furthermore, CRF of our population was assessed objectively. However, relative MPA did not perform well in our study. This was likely due to 2-min interruption allowance during the 10-min-bout data processing. Actilife defines interruptions as "minutes outside of the minimum and maximum count levels" [31]. These interruptions do not distinguish peaks from drops. Unlike the MVPA and VPA (with only drops counted as interruptions), the MPA threshold is defined as a range where peaks and drops crossing the range are added up, resulting in higher number of discounted bouts.

The extensive analysis of non-participants in our study revealed presence of a selection bias, with the participants reporting higher PA, education and better health than non-participants [19]. Our participants were likely fitter than non-participants. Nevertheless, our population was diverse and included healthy as well as older adults with co-morbidities [19]. Our population is a good representation of the general older Norwegian population, with similar co-morbidity prevalence as described in the 2015 Norwegian Institute of Public Health report [19, 32]. The thresholds for relative PA analysis were sex and CRF stratified, and created specifically for our population. However, the external generalizability of our findings to populations of different ages and ethnicities is limited, and the cross-sectional nature of our study prevents us from establishing causality. Therefore, longitudinal studies on more diverse populations with hard endpoints such as morbidity and mortality are needed.

\section{Conclusion}

In conclusion, higher proportion of our sample of older adults met relative versus absolute PA recommendation. Not meeting the relative PA recommendation associated with higher likelihood for having MetS, with no such association observed when not meeting the absolute PA recommendation. Since relative intensity is part of the current PA recommendation, it should be considered when assessing population PA and associated health risks.

\section{Additional file}

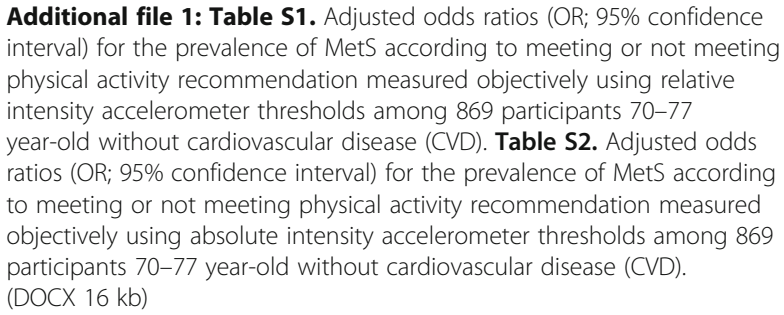
physical activity recommendation measured objectively using relative intensity accelerometer thresholds among 869 participants 70-77 year-old without cardiovascular disease (CVD). Table S2. Adjusted odds ratios (OR; 95\% confidence interval) for the prevalence of MetS according to meeting or not meeting physical activity recommendation measured objectively using absolute intensity accelerometer thresholds among 869 participants 70-77 year-old without cardiovascular disease (CVD). (DOCX $16 \mathrm{~kb})$

\section{Abbreviations}

CRF: Cardiorespiratory fitness; MetS: Metabolic syndrome; MPA: Moderate physical activity; MVPA: Moderate to vigorous physical activity; PA: Physical activity; VO2peak: Peak oxygen uptake; VPA: Vigorous physical activity

\section{Acknowledgements}

The cardiopulmonary tests were performed at the core facility NeXt Move, Norwegian University of Science and Technology. We thank the Clinical Research Facility at St. Olavs Hospital for outstanding assistance during the testing and all staff for the contribution to data collection.

\section{Funding}

The study was supported by grants from the K.G. Jebsen Foundation, the Norwegian Research Council, the Liaison Committee between the Central Norway Regional Health Authority and the Norwegian University of Science and Technology.

\section{Availability of data and materials}

Due to ethical restrictions involving participant consent, third party data used from the Generation 100 Study in research projects will when reasonably requested by others will be made available on request to the Generation 100 project leader Dorthe Stensvold. (dorthe.stensvold@ntnu.no). 


\section{Authors' contributions}

$\mathrm{NZ}$ and JN contributed equally to this work and should both be considered first authors. All authors contributed to the conception and design of the study. NZ, HV, NPA, TC, SBS and DS were responsible for the collection of the Generation 100 data in corporation with colleagues at the Cardiac Exercise Research Group at the Norwegian University of Science and Technology, Norway. NZ, JN and SBS provided the data for analysis and undertook the data analysis. NZ and JN drafted the manuscript. All authors provided critical insight and revisions to the manuscript. All authors read and approved the final version of the manuscript submitted for publication.

\section{Competing interests}

The funding organizations played no part in the design and execution of the study, in the data collection, analysis, and interpretation of the data or in the preparation, review, or approval of the or manuscript writing. There are no further disclosures to report and no conflicts of interest.

\section{Consent for publication}

Not applicable.

\section{Ethics approval and consent to participate}

All participants signed informed consent. The study was approved by the Regional Committee for Medical Research Ethics (2013/1609/REK Midt) and complied with the Declaration of Helsinki principles.

\section{Publisher's Note}

Springer Nature remains neutral with regard to jurisdictional claims in published maps and institutional affiliations.

\section{Author details}

${ }^{1}$ The K.G. Jebsen Center of Exercise in Medicine at Department of Circulation and Medical Imaging, Faculty of Medicine and Health Sciences, Norwegian University of Science and Technology, Trondheim, Norway. ${ }^{2}$ Department of Sociology and Political Science, Faculty of Social Sciences and Technology Management, Norwegian University of Science and Technology, Trondheim, Norway. ${ }^{3}$ Department of Cancer Research and Molecular Medicine, Faculty of Medicine and Health Sciences, Norwegian University of Science and Technology, Trondheim, Norway. ${ }^{4}$ Department of Nursing Science, Faculty of Medicine and Health Sciences, Norwegian University of Science and Technology, Trondheim, Norway. ${ }^{5}$ School of Human Movement \& Nutrition Sciences, University of Queensland, Brisbane, Australia.

Received: 22 June 2016 Accepted: 5 May 2017 Published online: 16 May 2017

\section{References}

1. GBD 2013. Global, regional, and national age-sex specific all-cause and cause-specific mortality for 240 causes of death, 1990-2013: a systematic analysis for the Global Burden of Disease Study 2013. Lancet (London, England). 2015;385(9963):117-71. doi:10.1016/s0140-6736(14)61682-2.

2. Nelson ME, Rejeski WJ, Blair SN, Duncan PW, Judge JO, King AC, et al. Physical activity and public health in older adults: recommendation from the American College of Sports Medicine and the American Heart Association. Circulation. 2007;116(9):1094-105. doi:10.1161/circulationaha.107.185650.

3. Strath SJ, Kaminsky LA, Ainsworth BE, Ekelund U, Freedson PS, Gary RA, et al. Guide to the assessment of physical activity: clinical and research applications: a scientific statement from the American Heart Association. Circulation. 2013;128(20):2259-79. doi:10.1161/01.cir.0000435708.67487.da.

4. Hagstromer M, Troiano RP, Sjostrom M, Berrigan D. Levels and patterns of objectively assessed physical activity-a comparison between Sweden and the United States. Am J Epidemiol. 2010;171(10):1055-64. doi:10. 1093/aje/kwq069.

5. Troiano RP, Berrigan D, Dodd KW, Masse LC, Tilert T, McDowell M. Physical activity in the United States measured by accelerometer. Med Sci Sports Exerc. 2008;40(1):181-8. doi:10.1249/mss.0b013e31815a51b3.

6. Actigraph. What are counts? 2017. https://actigraph.desk.com/customer/en/ portal/articles/2515580-what-are-counts-.

7. Freedson PS, Melanson E, Sirard J. Calibration of the Computer science and Applications, Inc. accelerometer. Med Sci Sports Exerc. 1998;30(5):777-81.
8. Evenson KR, Buchner DM, Morland KB. Objective measurement of physical activity and sedentary behavior among US adults aged 60 years or older. Prev Chronic Dis. 2012;9:E26.

9. Lee IM, Sesso HD, Oguma Y, Paffenbarger RS Jr. Relative intensity of physical activity and risk of coronary heart disease. Circulation. 2003;107(8):1110-6.

10. Kelly P, Murphy M, Oja P, Murtagh EM, Foster C. Estimates of the number of people in England who attain or exceed vigorous intensity exercise by walking at 3 mph. J Sports Sci. 2011;29(15):1629-34. doi:10.1080/02640414.2011.609897.

11. Myers J, Prakash M, Froelicher V, Do D, Partington S, Atwood JE. Exercise capacity and mortality among men referred for exercise testing. N Engl J Med. 2002;346(11):793-801. doi:10.1056/NEJMoa011858.

12. Shephard RJ. Absolute versus relative intensity of physical activity in a dose-response context. Med Sci Sports Exerc. 2001;33(6 Suppl):S400-18. discussion S19-20.

13. Ozemek C, Cochran HL, Strath SJ, Byun W, Kaminsky LA. Estimating relative intensity using individualized accelerometer cutpoints: the importance of fitness level. BMC Med Res Methodol. 2013;13:53. do:10.1186/1471-2288-13-53.

14. World Health Organization. Global recommendations on physical activity for health; 2010. p. 32. http://whqlibdoc.who.int/publications/2010/ 9789241599979_eng.pdf

15. Zisko N, Carlsen T, Salvesen O, Aspvik NP, Ingebrigtsen JE, Wisloff $U$, et al. New relative intensity ambulatory accelerometer thresholds for elderly men and women: the Generation 100 study. BMC Geriatr. 2015;15:97. doi:10. 1186/s12877-015-0093-1.

16. Alberti KG, Eckel RH, Grundy SM, Zimmet PZ, Cleeman JI, Donato KA, et al. Harmonizing the metabolic syndrome: a joint interim statement of the International Diabetes Federation Task Force on Epidemiology and Prevention; National Heart, Lung, and Blood Institute; American Heart Association; World Heart Federation; International Atherosclerosis Society; and International Association for the Study of Obesity. Circulation. 2009: 120(16):1640-5. doi:10.1161/CIRCULATIONAHA.109.192644.

17. Mozaffarian D, Kamineni A, Prineas RJ, Siscovick DS. Metabolic syndrome and mortality in older adults: the cardiovascular health study. Arch Intern Med. 2008;168(9):969-78. doi:10.1001/archinte.168.9.969.

18. Wen CJ, Lee YS, Lin WY, Huang HL, Yao CA, Sung PK, et al. The metabolic syndrome increases cardiovascular mortality in Taiwanese elderly. Eur J Clin Investig. 2008;38(7):469-75. doi:10.1111/j.1365-2362.2008.01965.x.

19. Stensvold D, Viken H, Rognmo O, Skogvoll E, Steinshamn S, Vatten $\sqcup$, et al. A randomised controlled study of the long-term effects of exercise training on mortality in elderly people: study protocol for the Generation 100 study. BMJ Open. 2015;5(2):e007519. doi:10.1136/bmjopen-2014-007519.

20. Wisloff U, Stoylen A, Loennechen JP, Bruvold M, Rognmo O, Haram PM, et al. Superior cardiovascular effect of aerobic interval training versus moderate continuous training in heart failure patients: a randomized study. Circulation. 2007;115(24):3086-94. doi:10.1161/circulationaha.106.675041.

21. Gibbons RJ, Balady GJ, Beasley JW, Bricker JT, Duvernoy WF, Froelicher VF, et al. ACC/AHA guidelines for exercise testing. A report of the American College of Cardiology/American Heart Association task force on practice guidelines (Committee on Exercise Testing). J Am Coll Cardiol. 1997;30(1):260-311.

22. Santos-Lozano A, Santin-Medeiros F, Cardon G, Torres-Luque G, Bailon R, Bergmeir C, et al. Actigraph GT3X: validation and determination of physical activity intensity cut points. Int J Sports Med. 2013;34(11):975-82. doi:10. 1055/s-0033-1337945.

23. Sun F, Norman IJ, While AE. Physical activity in older people: a systematic review. BMC Public Health. 2013;13:449. doi:10.1186/1471-2458-13-449.

24. Hall KS, Morey MC, Dutta C, Manini TM, Weltman AL, Nelson ME, et al. Activity-related energy expenditure in older adults: a call for more research. Med Sci Sports Exerc. 2014;46(12):2335-40.

25. Stata Corp. Bayesian analysis reference manual. 2015. http://www.stata.com/ manuals13/rbicnote.pdf\#rBICnote.

26. Miller NE, Strath SJ, Swartz AM, Cashin SE. Estimating absolute and relative physical activity intensity across age via accelerometry in adults. J Aging Phys Act. 2010;18(2):158-70.

27. Hansen BH, Holme I, Anderssen SA, Kolle E. Patterns of objectively measured physical activity in normal weight, overweight, and obese individuals (20-85 years): a cross-sectional study. PLoS One. 2013;8(1): e53044. doi:10.1371/journal.pone.0053044.

28. Hansen BH, Kolle E, Dyrstad SM, Holme I, Anderssen SA. Accelerometerdetermined physical activity in adults and older people. Med Sci Sports Exerc. 2012;44(2):266-72. doi:10.1249/MSS.0b013e31822cb354. 
29. Lohne-Seiler H, Hansen BH, Kolle E, Anderssen SA. Accelerometer-determined physical activity and self-reported health in a population of older adults (65-85 years): a cross-sectional study. BMC Public Health. 2014;14:284. doi:10.1186/1471-2458-14-284

30. Aspvik NP, Viken H, Zisko N, Ingebrigtsen JE, Wisloff U, Stensvold D. Are older adults physically active enough - a matter of assessment method? The Generation 100 study. PLoS One. 2016;11(11):e0167012. doi:10.1371/ journal.pone.0167012.

31. Actilife. How does bout detection work and where did we get our defaults? 2016. https://actigraph.desk.com/customer/en/portal/articles/2515834-howdoes-bout-detection-work-and-where-did-we-get-our-defaults-.

32. Norwegian Institute of Public Health. 2015. https://www.fhi.no/

Submit your next manuscript to BioMed Central and we will help you at every step:

- We accept pre-submission inquiries

- Our selector tool helps you to find the most relevant journal

- We provide round the clock customer support

- Convenient online submission

- Thorough peer review

- Inclusion in PubMed and all major indexing services

- Maximum visibility for your research

Submit your manuscript at www.biomedcentral.com/submit
C) Biomed Central 